\title{
Cytokine Adsorption Therapy during Extracorporeal Membrane Oxygenation in Adult Patients with COVID-19
}

\author{
Simão C. Rodeia Francisca Lopes Martins Philip Fortuna Luís Bento \\ Unidade de Urgência Médica (General ICU), Hospital de São José, Centro Hospitalar Universitário de Lisboa Central, \\ Lisbon, Portugal
}

\section{Keywords}

Blood purification - COVID-19. Cytokine release syndrome . Extracorporeal membrane oxygenation · Hemoadsorption . Interleukin-6

\begin{abstract}
Respiratory failure and systemic inflammation are paramount features of severe SARS-CoV-2 disease (COVID-19). Extracorporeal membrane oxygenation (ECMO) therapy has a potential role in patients with refractory disease. An inflammatory response due to blood contact with hemofilters, functioning as a synergic inflammatory stimulus, can lead to a hyperinflammatory state, relatable to cytokine release syndromes. After the first patient succumbed to a refractory vasodilatory shock believed to be due to hyperinflammatory state, a strategy of blood purification through cytokine adsorption therapy (CAT) with CytoSorb ${ }^{\circledR}$ was designed. In this case series, the authors describe the initial experience with such strategy. CAT was employed with no direct complications and helped controlling the inflammatory state, with all patients halting vasopressor support in $72 \mathrm{~h}$ and biomarker levels (C-reactive protein, ferritin, and interleukin-6) showing negative trends in most patients. Analysis of inflamma-
\end{abstract} related to the presence or absence of superinfection at the time of CAT implementation. In this case series of severe COVID-19 patients, 3 patients died - irreversible lung fibrosis, complications of critical hypoxemia before ECMO induction and complications of systemic anticoagulation were the causes. This case series aimed to contribute to the body of evidence substantiating CAT utilization in hyperinflammatory patients, namely, COVID-19 patients requiring ECMO rescue.

(c) 2021 S. Karger AG, Basel

\section{Introduction}

Systemic inflammation seems to be a prominent feature in COVID-19, leading to considerable damage in severe cases $[1,2]$. Descriptions seem to point out an elevation in pro-inflammatory cytokines, such as interleukin (IL)-2, IL-6, IL-10, interferon gamma, and TNF-alpha [1, $3]$, with concomitant activation of innate cellular immune response. Targeted cellular immune response may be blunted by virus-derived lymphopenia [4]. Some patients experience a hyperinflammatory state relatable to 
cytokine release syndromes (CRS, "cytokine storm") [3, $5]$, and a hypercoagulable state $[6,7]$, not classically observed in influenza patients [8]. Consequently, several centers and groups try different therapeutic approaches, while ongoing trails evaluate the efficacy of hydroxychloroquine, glucocorticoids, and drugs targeting IL-1, IL-6, IL-23a, and GM-CSF [9].

Throughout the world, cases series have described respiratory failure as the most frequent form of severe disease and the main cause of death in COVID-19 patients [10-14]. Where resources permit, extracorporeal membrane oxygenation (ECMO) has been used as a salvage therapy in selected cases [15-17]. One caveat to this approach is the possibility of inflammatory response amplification triggered by blood elements contacting the hemofilter $[18,19]$. To date, no strategy has proven efficacious in reducing the impact of ECMO-related systemic inflammation [20].

There is a rationale for the use of immunomodulation in an attempt to control pro-inflammatory response [21, 22]. While high-quality evidence precluding formal recommendation of blood purification techniques in hyperinflammatory states is lacking, experts consider it merits ponderation [22, 23]. In COVID-19, virus-activated CRS is linked to increased mortality, which invites the use of aggressive cytokine control therapies, such as hemoadsorption. The routine use of hemoadsorption with CytoSorb ${ }^{\circledR}$ has been described as safe in patients on ECMO support [24]. Aiming to share a favorable experience with cytokine adsorption therapy (CAT), the authors presented a case series where CAT was used alongside ECMO to control or prevent an overpowered inflammatory response.

\section{Case Series}

Patients in this case series were admitted to our ICU or referred to our care for extracorporeal respiratory support due to COVID-19-related respiratory failure ${ }^{1}$. All patients received standard supportive therapy - oxygen supplementation, invasive mechanical ventilation (IMV, using lung-protective ventilation), PEEP titration, neuromuscular blockade, and prone positioning (PP). Patients received other therapies according to the clinicians' decisions and local protocols. At the time of ECMO induc-

1 Our ICU is part of the first national reference centre for COVID-19 in the southern part of the country, as well as an Extracorporeal Life Support Organization (ELSO) Centre and a National Reference Centre for ECMO. tion, most had mechanical properties typical of restrictive pathology (ARDS). Table 1 details the most relevant parameters for each patient.

The hospital's clinical board authorized the use of CAT after the death of the first COVID-19 patient on ECMO support, $8 \mathrm{~h}$ after its initiation, due to refractory shock. All other causes for refractory shock were excluded (cardiac output was at $7 \mathrm{~L} / \mathrm{min}$, hemoglobin was stable, and cannulation site complications were excluded), and CRS aggravated by membrane exposure was deemed as the cause of shock. A strategy of systematic use of CAT for IL-6 levels $>100 \mathrm{pg} / \mathrm{mL}$ in ECMO patients was henceforth adopted. CAT was performed in parallel with ECMO circuit using 2 sequential CytoSorb ${ }^{\circledR}$ cartridges (each for $24 \mathrm{~h}$, according to manufacturer suggestion and previously described practice [24-27]).

\section{Patient 1}

A 29-year-old Bengali male with medicated arterial hypertension and obesity was admitted to another hospital, in the third week of fever, productive cough, dyspnea, myalgia, and asthenia. Early $(<24 \mathrm{~h})$ transfer to the ICU was needed for respiratory support. Bacterial superinfection was suspected and empiric antibacterial therapy started. During the 5 following days, he deteriorated developing severe ARDS with little response to neuromuscular blockade, prone positioning, and antibacterial therapy escalation. After $24 \mathrm{~h}$ with $\mathrm{Pa} / \mathrm{FiO}_{2}$ constantly $<100$, rescue therapy with nitrous oxide $(20 \mathrm{ppm}$ maximal dosing) was started but proved equally ineffective. With worsening respiratory acidosis limiting protective ventilation and a cardiac arrest episode secondary to tracheal tube obstruction during prone ventilation, ECMO rescue was decided. CAT was initiated from the start of extracorporeal support. The patient improved, allowing for a short ECMO run (6 days) but at sedation withdrawal, a super-refractory myoclonic status epilepticus was documented. Neuroimaging revealed hypoxic encephalopathy. Evoked potentials revealed absent N20 reflexes. Goals of care were readjusted toward comfort care and the patient was transferred to the ward, where he was cared for and offered palliative terminal sedation.

\section{Patient 2}

A 27-year-old healthy Bengali male presented to the emergency department with 4-day history of fatigue, fever, nonproductive cough, and dyspnea. Experiencing worsening respiratory failure, he was intubated and transferred to our ICU. For the first 5 days, the patient exhibited no respiratory distress, receiving low levels of pres- 
Table 1. Clinical characteristics of patients with COVID-19 treated with ECMO and CAT (Cytosorb $\left.{ }^{\oplus}\right)$

\begin{tabular}{|c|c|c|c|c|c|c|}
\hline & Ref & Patient 1 & Patient 2 & Patient 3 & Patient 4 & Patient 5 \\
\hline Age & Years & 29 & 27 & 43 & 68 & 58 \\
\hline Sex & & Male & Male & Male & Male & Male \\
\hline Conditions & - & HTN & None & None & None & HTN, T2DM, and CVD \\
\hline Medications & - & ACEI & None & None & None & Insulin \\
\hline Delay symptoms-IMV & Days & 21 & 5 & 31 & 4 & 7 \\
\hline Delay IMV-ECMO & Days & 6 & 5 & 7 & 7 & 0 \\
\hline Delay symptoms-Cytosorb ${ }^{\circledR}$ & Days & 27 & 10 & 39 & 11 & 7 \\
\hline Vasopressor & Days & 6 & 2 & 2 & 2 & 9 \\
\hline IMV & Days & 18 & 11 & 11 & 47 & 40 \\
\hline ECMO & Days & 5 & 5 & 8 & 38 & 25 \\
\hline Empiric antibacterial & - & Yes & Yes & Yes & Yes & Yes \\
\hline Hydroxychloroquine & - & Yes & Yes & Yes & No & No \\
\hline Vitamin B1 and vitamin C & - & Yes & Yes & No & No & No \\
\hline Antiviral & - & $\mathrm{LPV} / \mathrm{r}$ & $\mathrm{LPV} / \mathrm{r}$ & No & RDV & No \\
\hline Methylprednisolone & - & No & No & No & Yes & No \\
\hline NMB pre-ECMO & - & Yes & Yes & Yes & Yes & Yes \\
\hline Prone positioning pre-ECMO & - & Yes & Yes & Yes & Yes & Yes \\
\hline Worst $C_{\text {stat }}$ & $\begin{array}{l}\mathrm{mL} / \mathrm{cm} \\
\mathrm{H}_{2} \mathrm{O}\end{array}$ & 22 & 25 & 60 & 67 & 32 \\
\hline Worst $\mathrm{Pa} / \mathrm{FiO}_{2}$ & - & 73 & 111 & 70 & 92 & 50 \\
\hline
\end{tabular}

HTN, arterial hypertension; ACEl, angiotensin-converting enzyme inhibitor; T2DM, type 2 diabetes mellitus; CVD, cerebral vascular disease; IMV, invasive mechanical ventilation; ECMO, extracorporeal membrane oxygenation; LPV, lopinavir/ritonavir; RDV, remdesivir; NMB, neuromuscular blockade; $\mathrm{C}_{\text {stat }}$ static compliance; CAT, cytokine adsorption therapy.

sure support ventilation, despite high $\mathrm{FiO}_{2}$ needs. On the fifth day, however, he became febrile and tachypneic with progressive dyssynchrony and ensuing hypoxemia. $\mathrm{He}$ was deeply sedated, switched to controlled mechanical ventilation and $\mathrm{NMB}$ and PP were instituted. Respiratory failure was refractory and IMV parameters approached nonsecure levels, which led to implementation of ECMO and CAT (febrile state and moderately elevated IL-6 level). He received a short uneventful ECMO run (5 days), tolerating IMV discontinuation the day after ECMO withdrawal. He was transferred to Infectious Diseases ward, with no complications registered, notably infectious ones.

\section{Patient 3}

A 43-year-old healthy Eurocaucasian male was rescued under ECMO from another institution where he was admitted for COVID-19 respiratory failure and was initially managed noninvasively. At the seventh day of admission, clinical deterioration with fever and aggravating respiratory failure led to intubation and IMV. Prolonged NMB, various periods of $\mathrm{PP}$ and nitric oxide (10 ppm max) were tried with short lasting success, with worsening respira- tory mechanics precluding protective ventilation, and $\mathrm{Pa} /$ $\mathrm{FiO}_{2}$ under 100. ECMO rescue was arranged. Assuming bacterial infection as the cause for the deterioration and with IL-6 unavailable at the referring institution, CAT was not initiated from the start. For the first $6 \mathrm{~h}$ of ECMO therapy, a state of vasodilatory shock was seen with hypotension, elevated serum lactate and veno-arterial $\mathrm{PaCO}_{2}$ gap, leading to increasing noradrenaline requirements. Reflecting prior experience, CAT was initiated and a swift resolution of the shock state was observed. ECMO support allowed for respiratory improvement within 6 days. However, a state of severe hemorrhagic shock developed the eighth day due to a large retroperitoneal hemorrhage (related to subclinical disseminated intravascular coagulation), additionally complicated by perforated ischemic bowel. The patient then developed refractory septic shock and ultimately died, 42 days after initial hospital admission, and 27 days after ECMO induction.

\section{Patient 4}

A 68-year-old healthy Eurocaucasian male presented to the emergency department with headache and shortness of breath. Due to critical desaturation, he was imme- 


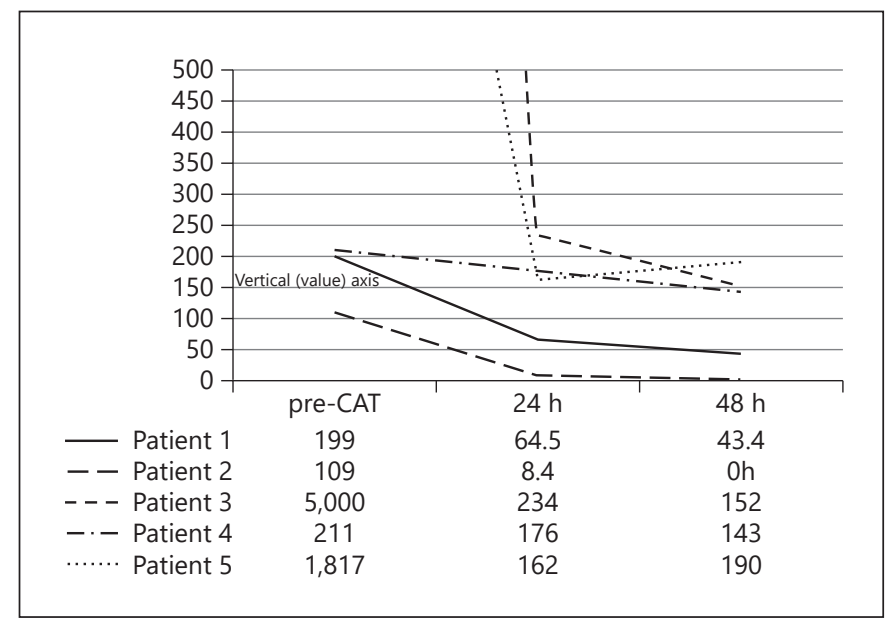

Fig. 1. IL-6 levels (pg/mL) evolution. IL, interleukin; CAT, cytokine adsorption therapy.

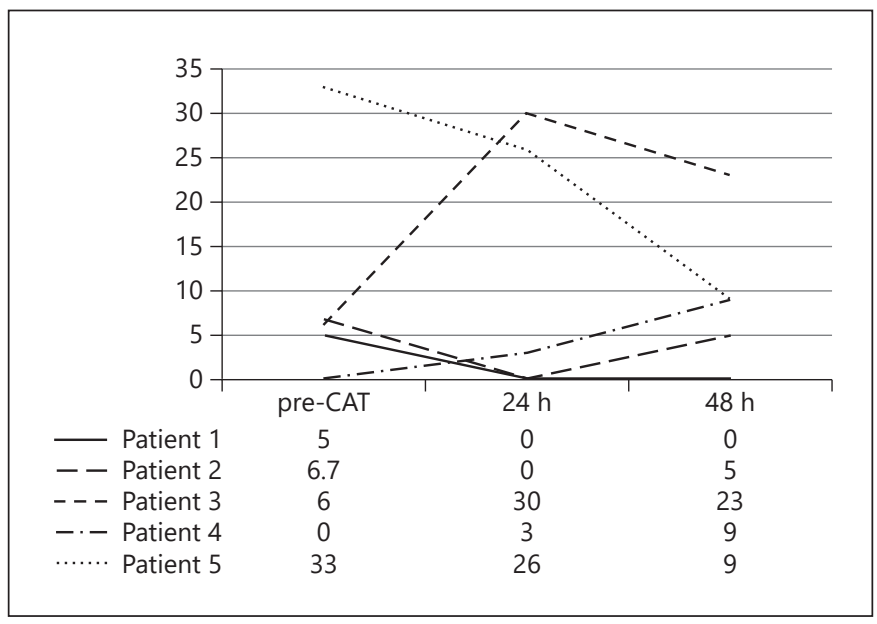

Fig. 2. NAD requirement ( $\mu \mathrm{g} / \mathrm{min})$ evolution. CAT, cytokine adsorption therapy; NAD, noradrenaline.
Fig. 3. Maximum variation of vasopressor support and inflammatory biomarkers after CAT. CAT, cytokine adsorption therapy; IL, interleukin; C-reactive protein; PCT, procalcitonin.

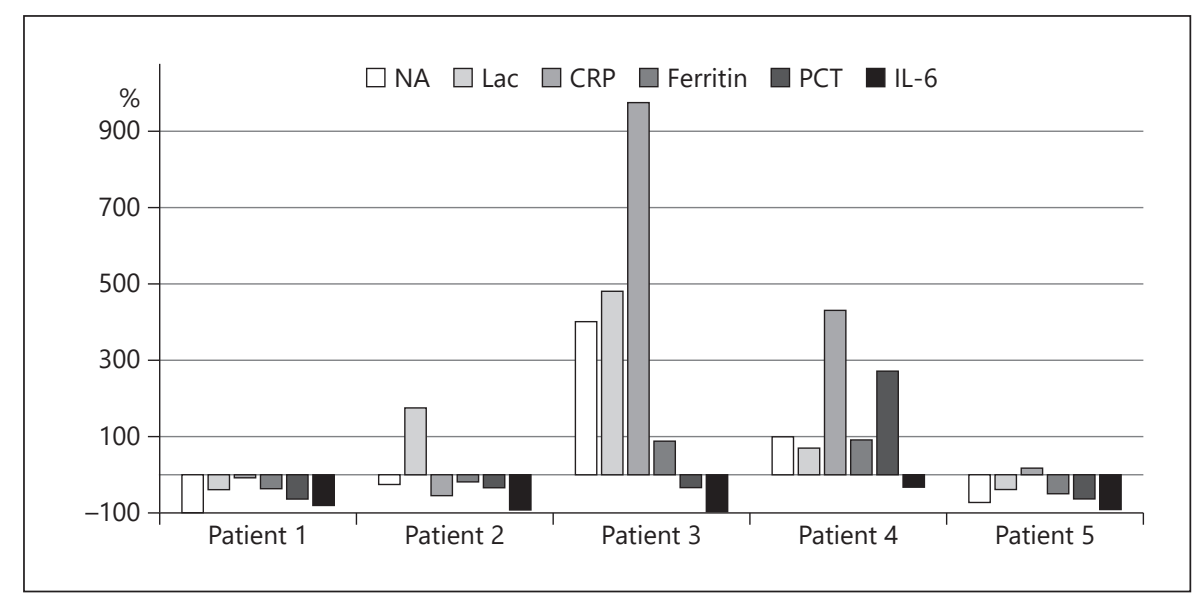

diately intubated and transferred to our ICU. Febrile with elevated C-reactive protein and low procalcitonin, he was started on methylprednisolone and antibacterial therapy, while waiting for microbiology. He presented high respiratory drive with need for deep sedation to reach adequate ventilatory synchronization. Respiratory failure persisted leading to early use of NMB and PP. On the seventh day, fever reappeared and respiratory acidemia became impossible to manage using protective ventilatory parameters, and ECMO/CAT support was started. The patient remained difficult to manage requiring considerable ECMO support and profound sedation. After volume status optimization, ECMO drainage compromise leads to high-flow ECMO initiation $(\mathrm{VV}-\mathrm{V})$. The patient sustained further clinical deterioration caused by 2 episodes of ventilatorassociated pneumonia, rendering the patient dependent on full ECMO support (blood flow $>5 \mathrm{~L} / \mathrm{min}$, sweep gas flow $>8 \mathrm{~L} / \mathrm{min}$ ) for several weeks. Ten days after clinical cure of the last infection, the patient remained ECMOdependent, respiratory mechanics suggested a stiff lung $\left(\mathrm{C}_{\text {stat }} 2-8 \mathrm{~mL} / \mathrm{cm} \mathrm{H}_{2} \mathrm{O}\right)$ and lung imaging revealed bilateral fibrosis in over seventy percent lung area. Assuming irreversible lung fibrosis due to repeated lung infections, with installed intensive care myopathy and long-standing deep sedation, the patient was considered noneligible for lung transplantation and further ECMO support was considered nonbeneficial. Support was withdrawn, allowing patient's death after 50 days of ICU treatment.

\section{Patient 5}

A 68-year-old black African male, with arterial hypertension, type 2 diabetes, and previous ischemic stroke, 
Table 2. Analytical evolution of patients with COVID-19 treated with ECMO and CAT (Cytosorb $\left.{ }^{\circledast}\right)$

\begin{tabular}{|c|c|c|c|c|c|c|c|c|c|c|c|c|}
\hline & \multicolumn{3}{|c|}{ Lactate, $\mathrm{mmol} / \mathrm{L}$} & \multicolumn{3}{|c|}{ CRP, mg/L } & \multicolumn{3}{|c|}{ Ferritin, ng/mL } & \multicolumn{3}{|c|}{$\mathrm{PCT}, \mathrm{ng} / \mathrm{mL}$} \\
\hline Patient 1 & 1 & 0.7 & 0.6 & 286 & 274 & 259 & 2,804 & 1,795 & 2,113 & 1.24 & 0.68 & 0.45 \\
\hline Patient 3 & 0 & 4.8 & 1.5 & 26 & 279 & 256 & $>2,000$ & 3,760 & 2,628 & 3.29 & 2.77 & 2.19 \\
\hline Patient 4 & 1.3 & 2.2 & 1.3 & 48 & 187 & 254 & 1,728 & 2,748 & 3,315 & 0.1 & 0.33 & 0.37 \\
\hline Patient 5 & 3 & 1.8 & 2 & 224 & 260 & 232 & 1,191 & 657 & 592 & 19.2 & 10.7 & 6.98 \\
\hline
\end{tabular}

CAT, cytokine adsorption therapy; CRP, C-reactive protein; PCT, procalcitonin; ELSO, Extracorporeal Life Support Organization; ECMO, extracorporeal membrane oxygenation.

was admitted to a district hospital due to COVID-19 pneumonia. Quick deterioration with refractory respiratory failure leads to ECMO rescue in $<24 \mathrm{~h}$. CAT was implemented for $48 \mathrm{~h}$ and a bacterial superinfection was suspected and treated empirically. He received a long ECMO run (29 days), due to multiple infectious complications (2 episodes of ventilator-associated pneumonia and a primary bacteremia). Throughout the ICU stay, he sustained hard to treat hyperactive delirium, causing prolonged ventilatory weaning (partially facilitated by a 4-day run of extracorporeal $\mathrm{CO}_{2}$ removal), alongside with severe tachypnea without pulmonary or systemic causes. After full respiratory support was discontinued, never fully recovering from an altered state of consciousness, the patient was transferred to Internal Medicine ward of the referring hospital 45 days after ICU admission.

\section{Discussion}

In this case series, all patients were managed with CAT alongside ECMO for suspected CRS or to prevent a hyperinflammatory state resulting from the synergistic proinflammatory stimuli from COVID-19 and ECMO. All had fever and elevated IL-6 prior to ECMO induction. It is however hard to diagnose CRS in COVID-19 patients with criteria being sometimes used too broadly, and a well-structured systematic review questioning whether the COVID-19 inflammatory organ dysfunction can be related to CRS [28]. The cause of the inflammatory state in these patients is probably explained by $>1$ mechanism (e.g., SARS-CoV-2 infection, bacterial superinfection, and hypoxic/hypotensive episodes). Such complexity casts a shadow over the potential benefit of CAT, inviting future studies with comparative analysis.

Nevertheless, CAT seems to have a role in controlling the inflammatory response, as judged by the reduction in
IL-6 and lactate levels and vasopressor requirements (Fig. 1-3). All patients suspended vasopressor support within $72 \mathrm{~h}$ of CAT start. In a case series using CAT to control inflammatory states purely caused by hyperinflammation [29], shock reversal and vasopressor support suspension were almost immediate. However, when CAT was used to control sepsis-related inflammation [24,27], a reduction in vasopressor requirements took longer to occur. IL-6 levels have been associated with increased severity and mortality in COVID-19 patients $[13,30]$. Two patients had IL-6 levels determined only after initiation of ECMO support. Patient 1, who received concomitant CAT from the onset of ECMO, had only moderately increased IL-6 values (199 pg/mL). Patient 3 had IL-6 level determined only at the time of arrival at our ICU, already under ECMO therapy. Despite IL-6 levels above the determination cutoff, CAT was not immediately started because of clinically suspected bacterial superinfection, and vasodilatory shock quickly ensued after perfusion through ECMO hemofilter commenced. Control of shock state with CAT despite infection persistence and continuing hemoperfusion through ECMO filter further points to a dysregulated inflammatory state.

Looking at other inflammatory biomarkers (Table 2), all patients had elevated ferritin and most had elevated C-reactive protein, with levels oscillating in nonspecific manner after ECMO/CAT initiation. Patient 4 exhibited increase in inflammatory markers after ECMO/CAT, with a respiratory fungal infection becoming symptomatic in subsequent days. Despite only small-to-modest elevations in procalcitonin (with exception of patients 3 and 5), most patients were shown, or at least suspected, to have bacterial superinfection surrounding the time of $\mathrm{ECMO} / \mathrm{CAT}$ initiation. Evaluating the maximal variation in vasopressor requirement and laboratory values (Fig. 3), 2 patients ( 3 and 4 ) exhibit a different profile with increase in inflammatory biomarkers despite CAT. This 
probably highlights an inflammatory state mainly due to superinfection, as opposite of patients 1,2 , and 5 , who present a clear suppression of inflammatory biomarkers with CAT and probably represent cases of inflammation mostly related to COVID-19.

As in previous series, patients sustained no direct complications of CAT, namely, severe thrombocytopenia. Two of nonsurviving patients sustained ulterior infections, one of them dying in result of refractory nosocomial septic shock and the other dying from irreversible lung fibrosis. Despite some sustained interest in anti-IL-6 therapy in COVID-19 patients [1,31-33], our group chose CytoSorb ${ }^{\circledR}$ for the potential added benefit in controlling ECMO-related systemic inflammation and the time limited effect, which improves infectious risk.

Our analysis presents with considerable limitations inherent to the retrospective nature of the report. The absence of control group forbids clear conclusions on efficacy, safety, and total organ dysfunction burden amelioration. The authors believe the path ahead for hemoadsorption must include higher quality evidence larger case-control series and well-designed clinical trials, in patients with septic shock and other hyperinflammatory states.

\section{Conclusion}

Despite the lack of solid RCT data proving their worth, pathophysiological rationale behind CAT and clinical evidence available in published case series and some lowquality evidence support its use. This report adds to this body of work. CAT seems a safe therapy and albeit expensive, its costs may be offset by its efficacy in controlling superinflammatory responses seen in COVID-19 patients, often augmented by the hemoperfusion through extracorporeal oxygenation filters. Long-term mortality and secondary infection rates need to be evaluated by larger series or controlled trials.

\section{Acknowledgments}

The authors would like to acknowledge the contribution of Pedro Eduardo Silva MD to the data presentation.

\section{Statement of Ethics}

Ethics Committee authorization was not sought as intervention was performed as clinically decided and data collection was retrospective, not with investigational purposes. Written informed consent for publication of this case report and any accompanying images was obtained from the patients or next of kin (1 deceased patient's family was unreachable).

\section{Conflict of Interest Statement}

The authors have no conflicts of interest to declare.

\section{Funding Sources}

There are no funding sources to declare.

\section{Author Contributions}

S.C.R. and F.L.M. drafted the manuscript. P.F. and L.B. critically reviewed the draft. All authors approved the submission.

\section{Data Availability Statement}

The datasets generated during and/or analysed during the current study are available from the corresponding author on reasonable request.

\section{References}

1 Tay MZ, Poh CM, Rénia L, MacAry PA, Ng LFP. The trinity of COVID-19: immunity, inflammation and intervention. Nat Rev Immunol. 2020;20(6):363-74.

$2 \mathrm{Xu} \mathrm{Z}$, Shi L, Wang Y, Zhang J, Huang L, Zhang C, et al. Pathological findings of COVID-19 associated with acute respiratory distress syndrome. Lancet Respir Med. 2020 Apr; 8(4):420-2.

3 Henderson LA, Canna SW, Schulert GS, Volpi S, Lee PY, Kernan KF, et al. On the alert for cytokine storm: immunopathology in COVID-19. Arthritis Rheumatol. 2020 Jul 10; 72(7):1059-63.
4 Gu J, Gong E, Zhang B, Zheng J, Gao Z, Zhong $Y$, et al. Multiple organ infection and the pathogenesis of SARS. J Exp Med. 2005; 202(3):415-24.

5 Mehta P, McAuley DF, Brown M, Sanchez E, Tattersall RS, Manson JJ. COVID-19: consider cytokine storm syndromes and immunosuppression. Lancet. 2020;395(10229):10334.

6 Becker RC. COVID-19 update: covid-19-associated coagulopathy. J Thromb Thrombolysis. 2020;50(1):54-67.

7 Thachil J, Tang N, Gando S, Falanga A, Cattaneo $\mathrm{M}$, Levi M, et al. ISTH interim guidance on recognition and management of coagulopathy in COVID-19. J Thromb Haemost. 2020; 18(5):1023-6.

8 Tang X, Du RH, Wang R, Cao TZ, Guan LL, Yang CQ, et al. Comparison of hospitalized patients with ARDS caused by COVID-19 and H1N1. Chest. 2020;158(1):195-205.

9 WHO. No Title. International Clinical Trials Registry Platform. [Online].

10 Ruan Q, Yang K, Wang W, Jiang L, Song J. Clinical predictors of mortality due to COVID-19 based on an analysis of data of 150 patients from Wuhan, China. Intensive Care Med. 2020;46(5):846-8. 
11 Zhou F, Yu T, Du R, Fan G, Liu Y, Liu Z, et al. Clinical course and risk factors for mortality of adult inpatients with COVID-19 in Wuhan, China: a retrospective cohort study. Lancet. 2020;395(10229):1054-62.

12 Grasselli G, Pesenti A, Cecconi M. Critical care utilization for the COVID-19 outbreak in Lombardy, Italy. JAMA. 2020 Apr 28;323(16): 1545-6.

13 Huang C, Wang Y, Li X, Ren L, Zhao J, Hu Y, et al. Clinical features of patients infected with 2019 novel coronavirus in Wuhan, China. Lancet. 2020 Feb;395(10223):497-506.

14 Zhang B, Zhou X, Qiu Y, Song Y, Feng F, Feng $\mathrm{J}$, et al. Clinical characteristics of 82 cases of death from COVID-19. PLoS One. 2020; 15(7):e0235458.

15 Kowalewski M, Fina D, Słomka A, Raffa GM, Martucci G, Lo Coco V, et al. COVID-19 and ECMO: the interplay between coagulation and inflammation - a narrative review. Crit Care. 2020;24(1):1-10.

16 Li M, Gu SC, Wu XJ, Xia JG, Zhang Y, Zhan QY. Extracorporeal membrane oxygenation support in 2019 novel coronavirus disease: indications, timing, and implementation. Chin Med J. 2020 May;133(9):1115-7.

17 Ramanathan K, Antognini D, Combes A, Paden M, Zakhary B, Ogino M, et al. Planning and provision of ECMO services for severe ARDS during the COVID-19 pandemic and other outbreaks of emerging infectious diseases. Lancet Respir Med. 2020;8(5):518-26.

18 Burrell AJC, Lubnow M, Enger TB, Nanjayya VB, Philipp A, Malfertheiner MV, et al. The impact of venovenous extracorporeal membrane oxygenation on cytokine levels in patients with severe acute respiratory distress syndrome: a prospective, observational study. Crit Care Resusc. 2017 Oct;19(Suppl 1):3744.
19 Al-Fares A, Pettenuzzo T, Del Sorbo L. Extracorporeal life support and systemic inflammation. Intensive Care Med Exp. 2019;7(Suppl 1):46-14.

20 Landis RC, Brown JR, Fitzgerald D, Likosky DS, Shore-Lesserson L, Baker RA, et al. Attenuating the systemic inflammatory response to adult cardiopulmonary bypass: a critical review of the evidence base. J Extra Corpor Technol. 2014 Sep;46(3):197-211.

21 RECOVERY Collaborative Group; Horby P, Lim WS, Emberson JR, Mafham M, Bell JL, et al. Dexamethasone in hospitalized patients with covid-19 - preliminary report. N Engl J Med. 2021 Feb 25;384(8):693-704.

22 Angus DC, van der Poll T. Severe sepsis and septic shock. N Engl J Med. 2013 Aug 29; 369(9):2063-51.

23 Ronco C, Reis T, De Rosa S. Coronavirus epidemic and extracorporeal therapies in intensive care: si vis pacem para bellum. Blood $\mathrm{Pu}$ rif. 2020;49(3):255-8.

24 Akil A, Ziegeler S, Reichelt J, Rehers S, Abdalla O, Semik M, et al. Combined use of cytosorb and ECMO in patients with severe pneumogenic sepsis. Thorac Cardiovasc Surg. 2021 Apr;69(3):246-51.

25 CytoSorbents. CytoSorb therapy indications and set-up [Internet]. 2016. Available from: http: //cytosorb-therapy.com/wp-content/ uploads/2016/03/CytoSorb_Booklet_ EN_1.0.pdf.

26 Scheier J, Humbert V; CytoSorbents. Options for actions regarding the use of CytoSorb therapy in COVID-19 patients (Status April 8th 2020). 2020. p. 1-5.
27 Kogelmann K, Jarczak D, Scheller M, Drüner M. Hemoadsorption by cytosorb in septic patients: a case series. Crit Care. 2017;21(1):7410.

28 Leisman DE, Ronner L, Pinotti R, Taylor MD, Sinha P, Calfee CS, et al. Cytokine elevation in severe and critical COVID-19: a rapid systematic review, meta-analysis, and comparison with other inflammatory syndromes. Lancet Respir Med. 2020 Dec;8(12):1233-44.

29 Träger K, Fritzler D, Fischer G, Schröder J, Skrabal C, Liebold A, et al. Treatment of postcardiopulmonary bypass SIRS by hemoadsorption: a case series. Int J Artif Organs. 2016 Mar 25;39(3):141-6.

30 Chen X, Zhao B, Qu Y, Chen Y, Xiong J, Feng $\mathrm{Y}$, et al. Detectable serum severe acute respiratory syndrome coronavirus 2 viral load (RNAemia) is closely correlated with drastically elevated interleukin 6 level in critically ill patients with coronavirus disease 2019. Clin Infect Dis. 2020 Nov 5;71(8):1937-42.

31 Poston JT, Patel BK, Davis AM. Management of critically ill adults with COVID-19. JAMA. 2020;323(18):1839-41.

32 Berlot G, Pintacuda S, Moro E, Paluzzano G, Scamperle A, Chillemi A, et al. Effects of tocilizumab versus hemoadsorption combined with tocilizumab in patients with SARSCoV-2 pneumonia: preliminary results. Int $J$ Artif Organs. 2021 Feb 11;39139882198933.

33 Toniati P, Piva S, Cattalini M, Garrafa E, Regola F, Castelli F, et al. Tocilizumab for the treatment of severe COVID-19 pneumonia with hyperinflammatory syndrome and acute respiratory failure: a single center study of 100 patients in Brescia, Italy. Autoimmun Rev. 2020 Jul;19(7):102568. 